\title{
Dispepsia Funcional: um estudo epidemiológico e clínico
}

\section{Functional dyspepsia: a clinical and epidemiological study}

Dispepsia funcional: un estudio clínico y epidemiológico

Wianne Santos Silva

ORCID: https://orcid.org/0000-0001-8006-8934

Universidade Tiradentes, Brasil

E-mail: wiannessilva@gmail.com

Gabriel Ponciano Santos de Carvalho

ORCID: https://orcid.org/0000-0001-7476-7907

Universidade Tiradentes, Brasil

E-mail: gabriel.ponciano19@gmail.com

Anna Marcela Lima Fonseca

ORCID: https://orcid.org/0000-0001-5519-965X

Universidade Tiradentes, Brasil

E-mail: marcela.lima0102@gmail.com

Kellyn Mariane Souza Sales

ORCID: https://orcid.org/0000-0001-6233-5103

Universidade Tiradentes, Brasil

E-mail: kellynsales1@gmail.com

Giovanna Pimentel Oliveira Silva

ORCID: https://orcid.org/0000-0002-4981-8466 Universidade Tiradentes, Brasil

E-mail: giovannapimentel96@gmail.com

Thaissa Carvalho Viaggi

ORCID: https://orcid.org/0000-0002-0026-7149 Universidade Tiradentes, Brasil

E-mail: thaissaviaggi@gmail.com

Beatriz Carvalho Aragão

ORCID: https://orcid.org/0000-0002-2805-9675

Universidade Tiradentes, Brasil

E-mail: bilaaragaoc@gmail.com

Ana Monize Ribeiro Fonseca

ORCID: https://orcid.org/0000-0002-7565-8435 Universidade Tiradentes, Brasil

E-mail: anamonizerf@gmail.com

Allef Francisco Lira da Rocha Braga

ORCID: https://orcid.org/0000-0001-7326-0012

Universidade Tiradentes, Brasil

E-mail: allefbraga@gmail.com

Anna Sophia Almeida Gouveia

ORCID: https://orcid.org/0000-0001-6019-4204

Universidade de Santa Cruz do Sul, Brasil

E-mail: annagoweia@gmail.com

Sender Jankiel Miszputen

ORCID: https://orcid.org/0000-0003-4487-5004

Universidade Federal de São Paulo, Brasil

E-mail: sender.miszputen@unifesp.br

Leda Maria Delmondes Freitas Trindade

ORCID: https://orcid.org/0000-0003-4300-4274

Universidade Tiradentes, Brasil

E-mail: ledeltrin@gmail.com

\begin{abstract}
Resumo
Introdução: A dispepsia funcional é uma síndrome gastrointestinal desafiadora pelos diversos fatores que podem gerála e pelos critérios exigidos para diagnosticá-la. Entretanto, é possível entender o perfil epidemiológico e clínico de seus portadores, bem como identificar a influência de algumas condições em suas manifestações. Objetivo: avaliar a prevalência da dispepsia funcional e caracterizar os aspectos epidemiológicos e clínicos. Metodologia: estudo prospectivo, transversal, tipo survey inquérito, com pacientes dispépticos encaminhados ao serviço de endoscopia digestiva alta. A amostra foi composta por 859 indivíduos com diagnóstico clínico de dispepsia, sendo aplicados questionários autoexplicativos: sociodemográfico, Inventário de Ansiedade de Beck, Inquérito de Depressão de Beck
\end{abstract}


e o questionário de ROMA III. Resultados: do total de entrevistados, 36 (4,19\%) preencheram os critérios ROMA IV para dispepsia funcional, que engloba duas síndromes clínicas diferentes: a síndrome do desconforto pós-prandial e a síndrome da dor epigástrica. A faixa etária média foi 31,5 anos (19,2-39,7), 23 (63,9\%) eram procedentes de Aracaju, $32(88,8 \%)$ do sexo feminino, $21(60 \%)$ solteiros e $19(54,3 \%)$ consideravam-se de cor parda. Foram identificados 19 $(52,8 \%)$ pacientes portadores de ansiedade e $9(25 \%)$ com depressão. Segundo os critérios, 10 (27,8\%) apresentaram síndrome do desconforto pós-prandial, $07(19,4 \%)$ a síndrome da dor epigástrica e $19(52,8 \%)$ os dois subtipos clínicos simultaneamente. Conclusão: a dispepsia funcional foi mais prevalente entre as mulheres, indivíduos solteiros, cor parda e portadores de ansiedade. Não houve significância estatística quanto ao tipo de apresentação clínica, embora a manifestação concomitante dos dois subtipos, tenha sido mais prevalente.

Palavras-chave: Dispepsia; Epidemiologia; Sinais e sintomas.

\begin{abstract}
Introduction: Functional dyspepsia is a challenging gastrointestinal syndrome due to the various factors that may generate it and the criteria required to diagnose it. However, it is possible to understand the epidemiological and clinical profile of these patients, as well as to identify the influence of some conditions on their manifestations. Objective: to assess the prevalence of functional dyspepsia and its characterize the epidemiological and clinical aspects. Methodology: prospective, cross-sectional, survey-type study, with dyspeptic patients referred to the upper digestive endoscopy service. The sample consisted of 859, with a clinical diagnosis of dyspepsia, and self-explanatory questionnaires were reported: sociodemographic, Beck Anxiety Inventory, Beck Depression Survey and the ROMA III questionnaire. Results: 36 (4.19\%) of the total respondents met ROMA IV criteria for functional dyspepsia, which encompasses two different clinical syndromes: postprandial discomfort syndrome and epigastric pain syndrome. The average age group was 31.5 years old (19.2-39.7), 23 (63.9\%) were from Aracaju, 32 (88.8\%) were female, 21 (60\%) were single and $19(54.3 \%)$ considered themselves brown. We identified $19(52.8 \%)$ patients with anxiety and 9 (25\%) with depression. According to the criteria, 10 (27.8\%) postprandial discomfort syndrome, 07 (19.4\%) epigastric pain syndrome and $19(52.8 \%)$ both simultaneous clinical subtypes. Conclusion: a functional dyspepsia was more prevalent among women, single dependents, brown skin color and people with anxiety. There was no statistical significance regarding the type of clinical presentation, although the simultaneous manifestation of the two subtypes was more prevalent.
\end{abstract}

Keywords: Dyspepsia; Epidemiology; Signs and symptoms.

\title{
Resumen
}

Introducción: La dispepsia funcional es un síndrome gastrointestinal desafiante debido a los diversos factores que pueden generarlo y los criterios necesarios para diagnosticarlo. Sin embargo, es posible comprender el perfil epidemiológico y clínico de sus pacientes, así como identificar la influencia de algunas afecciones en sus manifestaciones. Objetivo: evaluar la prevalencia de dispepsia funcional y caracterizar los aspectos epidemiológicos y clínicos. Metodología: estudio prospectivo, transversal, tipo encuesta, con pacientes dispépticos remitidos al servicio de endoscopia digestiva alta. La muestra estuvo conformada por 859, con diagnóstico clínico de dispepsia, y se reportaron cuestionarios autoexplicativos: sociodemográfico, Inventario de Ansiedad de Beck, Encuesta de Depresión de Beck y cuestionario ROMA III. Resultados: 36 (4,19\%) del número total de encuestados cumplieron los criterios ROMA IV para dispepsia funcional, que engloba dos síndromes clínicos diferentes: síndrome de malestar posprandial y síndrome de dolor epigástrico. El grupo de edad promedio fue de 31,5 años (19,2-39,7), 23 (63,9\%) eran de Aracaju, $32(88,8 \%)$ eran mujeres, $21(60 \%)$ eran solteras y $19(54,3 \%)$ se consideraban morenos. Identificamos $19(52,8 \%)$ pacientes con ansiedad y $9(25 \%)$ con depresión. Según los criterios, $10(27,8 \%)$ síndrome de malestar posprandial, 07 (19,4\%) síndrome de dolor epigástrico y 19 (52,8\%) ambos subtipos clínicos simultáneos. Conclusión: la dispepsia funcional fue más prevalente entre mujeres, solteros dependientes, piel morena y personas con ansiedad. No hubo significación estadística con respecto al tipo de presentación clínica, aunque la manifestación concomitante de los dos subtipos fue más prevalente.

Palabras clave: Dispepsia; Epidemiología; Signos y síntomas.

\section{Introdução}

Dispepsia Funcional (DF) é uma doença, de etiologia multifatorial, cujas queixas clínicas se localizam na parte superior do abdômen, com manifestação periódica podendo cronificar. Possui sinais e sintomas característicos como: dor abdominal, saciedade precoce, plenitude gástrica pós prandial e azia, com a ausência de uma causa orgânica identificável por meios diagnósticos convencionais (Vinaccia, Quiceno, Medina, Castañeda, \& Castelblanco, 2014).

A endoscopia digestiva alta (EDA) é um procedimento que permite a avaliação e análise direta do trato digestivo superior e possibilita o diagnóstico diferencial entre as dispepsias orgânica e funcional (Ferri, 2019). De acordo com os 
critérios de Roma IV, além de apresentar um ou mais sintomas gastrointestinais, no diagnóstico da DF deve-se excluir alterações estruturais (inclusive na EDA) que justifiquem o quadro clínico (Stanghellini et al., 2016; Santos, 2019).

Os critérios de Roma IV classificam o quadro clínico da DF em dois subtipos de acordo com os sintomas mais prevalentes: a síndrome do desconforto pós-prandial (SDPP) e a síndrome da dor epigástrica (SDE). A SDPP se refere à presença de sensação incômoda de desconforto após uma refeição habitual e de saciedade precoce, que pode interferir no seu término, enquanto a SDE é a presença de dor ou queimação em região epigástrica. Apesar de a subdivisão ter por objetivo auxiliar o manejo terapêutico da doença, não significa que uma queixa deva necessariamente ocorrer de forma isolada da outra, visto que as duas podem aparecer concomitantemente (Coelho et al., 2018).

Dentre os inúmeros diagnósticos diferenciais que existem para pessoas que apresentem sintomas como saciedade precoce, desconforto pós prandial e dor ou queimação epigástrica, a dispepsia não ulcerosa ou funcional é a mais frequente e afeta cerca de um quarto da população mundial. Esse distúrbio é responsável por cerca de 3\% a 5\% das consultas de cuidados primários em saúde e, apesar de ser uma problemática muito comum na saúde coletiva, ainda possui uma baixa procura pela assistência médica, sendo que apenas 8\% dos doentes procuram atendimento médico. (Ferreira, Bello, \& Gomes, 2018; Ferri, 2019).

Apesar de possuir uma fisiopatologia complexa e sem etiologia específica, a DF pode ser entendida como uma associação entre alterações do funcionamento do sistema imune e nervoso do organismo e as repercussões na motilidade gastroduodenal, principalmente quando ligado ao eixo "cérebro-intestino" (Drossman, 2016). Pelas interferências autonômicas eferentes ou pelo desequilíbrio do eixo neuroendócrino, hipotálamo-hipófise-adrenal, os fatores emocionais e psicológicos, atuam nos receptores do sistema nervoso entérico com repercussão sobre o esvaziamento gástrico, peristaltismo intestinal e as secreções digestivas, o que provoca o surgimento de sintomas gastrointestinais em diferentes segmentos do canal alimentar. A secreção anormal de hormônios liberadores de corticotrofina responde por alteração nos níveis de sensibilidade à dor periférica, o que pode explicar seu aumento em situações de estresse, em consequência ao aumento do cortisol (Mayer, Labus, Tillisch, Cole, \& Baldi, 2015; Moloney et al., 2016).

A DF é reconhecidamente desafiadora, no que diz respeito à estimativa da sua prevalência, tanto pela baixa procura da assistência primária pelo paciente portador de sintomas, quanto pelos critérios diagnósticos variáveis utilizados na maioria dos estudos sobre o tema e pela sua etiologia mal definida. Além disso, a síndrome dispéptica possui fatores de riscos importantes para sua manifestação, como o sexo feminino, a idade e a infecção por Helicobacter pylori (Harer \& Hasler, 2020).

Este estudo representa parte de um projeto sobre dispepsia, que teve como proposta avaliar a prevalência da Dispepsia Funcional, caracterizar os aspectos epidemiológicos e clínicos em portadores de sintomas dispépticos, submetidos a endoscopia digestiva alta, em duas clínicas privadas do município de Aracaju, Sergipe.

\section{Metodologia}

Trata-se de um estudo prospectivo, transversal, analítico, descritivo, do tipo survey inquérito sobre dispepsia funcional (DP), iniciado no ano de 2015 em Aracaju, Sergipe, Brasil. Em 2018 o projeto inicial passou por modificações na sua construção metodológica. De setembro de 2018 até dezembro de 2019 realizou-se nova coleta de dados em duas clínicas privadas de endoscopia digestiva. A amostra do tipo probabilística por conveniência foi composta por 859 indivíduos encaminhados ao serviço de endoscopia digestiva alta (EDA), com diagnóstico clínico de dispepsia.

Os exames endoscópico e histopatológico foram realizados por profissionais previamente definidos para manter o mesmo padrão de avaliação. Considerou-se como critérios de inclusão indivíduos maiores de 20 anos, de ambos os sexos, com 
diagnóstico clínico de dispepsia e ausência de lesão estrutural nos laudos endoscópicos e histopatológicos, e os de exclusão, mulheres grávidas, indivíduos com antecedentes de doenças físicas ou mentais limitantes ao processo de compreensão para a aplicação dos questionários. Todos os participantes foram abordados pelos pesquisadores, na sala de espera, antes de serem submetidos a EDA. Os laudos endoscópicos eram entregues aos pacientes após finalizado o exame. Os pesquisadores tinham acesso imediato aos mesmos e, posteriormente, ao laudo histopatológico, mediante autorização prévia, ao assinarem o Termo de Consentimento Livre e Esclarecido. Os entrevistados foram codificados para posterior contato e identificação dos laudos histopatológicos.

Antes da realização da endoscopia foram aplicados, sob a orientação dos pesquisadores, quatro questionários autoexplicativos com perguntas abertas e fechadas: questionário sociodemográfico e clínico, Inventários de Ansiedade (BAI) e de depressão de Beck (BDI) e o questionário de ROMA III. Para identificar o perfil sociodemográfico e clínico definiu-se como variáveis: idade, procedência, sexo, estado civil, cor da pele, hábitos sociais e antecedentes de saúde.

O Inventário de Ansiedade de Beck (BAI), composto por 21 questões de múltipla escolha, avalia a gravidade de sintomas comuns à ansiedade. As respostas quanto a caracterização de sintomas ansiosos podem ser: 0 para ausentes, 1 suaves, 2, moderados e 3 para forma severa. A presença de ansiedade foi estimada a partir do somatório das respostas: 0 a 10 não como ausência de sintomas ansiosos, 11 a 63 identificando algum grau de ansiedade.

O Inventário de Depressão de Beck (BDI), composto por 21 questões de múltipla escolha, contendo 4 itens cada uma, e pontuação de $0,1,2$ e 3, buscou medir a severidade do quadro depressivo. Os pontos de corte utilizados foram: entre 0 e 13 depressão mínima ou ausência, entre 14 e 19 leve, entre 20 e 28 moderada e acima de 28 como depressão severa.

Considerando que, em 2016, foram publicados os Critérios de ROMA IV, e que, até o início da nova versão deste projeto ainda não havia um novo questionário validado em português, optou-se por utilizar o questionário de ROMA III (Von Reisswitz, Mazzoleni, Sander, \& Francisconi, 2010) acrescentando as atualizações conceituais do ROMA IV para as variáveis dependentes: existência de plenitude pós-prandial e saciedade precoce para caracterizar a Síndrome do Desconforto Pós Prandial (SDPP) e a dor e/ou queimação epigástrica e azia para caracterizar a Síndrome da Dor Epigástrica (SDE).

A SDPP foi considerada quando o paciente referiu queixa de saciedade precoce e empachamento após uma refeição de tamanho habitual, respondidas nos itens 03, 04, 05 e 06 do questionário. A SDE foi considerada quando o paciente referiu queixa de desconforto ou dor em queimação em epigástrio, equivalentes aos itens 01,02 e de 07 a 13.

Utilizou-se o software R Core Team 2020. As variáveis categóricas foram descritas por meio de frequência absoluta e relativa percentual e, as variáveis contínuas, por mediana e intervalo quartil. A hipótese de independência entre variáveis categóricas foi testada pelos testes Qui-Quadrado de Pearson ou Exato de Fisher, e as diferenças entre medianas foram testadas pelo teste de Mann-Whitney. O nível de significância adotado foi de 5\%. Estudo aprovado pelo CEP/UNIT, parecer $\mathrm{n}^{\circ}$ 2.832.329.

\section{Resultados}

Do total de 859 entrevistados que apresentavam sintomas dispépticos, $766(89,1 \%)$ tinham alterações endoscópicas, $726(84,4 \%)$ alterações histopatológicas e $36(4,19 \%)$ pacientes não apresentaram lesão estrutural, tanto do ponto de vista, macro quanto microscópico, incluindo a pesquisa de H. pylori negativa na biópsia, preenchendo portanto, os critérios para o diagnóstico de dispepsia funcional, segundo Critérios de Roma III e IV.

A faixa etária mediana dos portadores de dispepsia funcional foi de 31,5 anos (19,2-39,7) sendo estatisticamente significativo $(p=0,002)$ o predomínio do sexo feminino, representando $32(88,8 \%)$ da amostra total $(\mathrm{p}=0,002)$. Eram 
procedentes de Aracaju 23 (63,9\%), 21 (60\%) solteiros e 19 (54,3\%) se consideravam de cor parda. Faziam uso de bebidas alcoólicas $5(13,9 \%)$ participantes e $20(58,8 \%)$ possuíam ensino médio completo (Tabela 1$)$.

Tabela 1. Aspectos sociodemográficos dos dispépticos funcionais. Aracaju 2018-2019.

\begin{tabular}{|c|c|c|c|c|}
\hline & & DISPEPSIA & NCIONAL & \\
\hline & $\begin{array}{l}\text { Válidos } \\
\text { n }(\%)\end{array}$ & $\underset{(n=36)}{\operatorname{Sim}}$ & $\begin{array}{c}\text { Não } \\
(\mathbf{n}=824)\end{array}$ & p-valor \\
\hline Idade, mediana (IIQ) & $859(99,9)$ & $31,5(19,2-39,7)$ & $37,0(30-46)$ & $<0,001^{\mathrm{V}}$ \\
\hline PROCEDÊNCIA, $n(\%)$ & & & & \\
\hline Aracaju & $627(72,9)$ & $23(63,9)$ & $604(73,3)$ & $0,467 \mathrm{Q}$ \\
\hline Interior de Sergipe & $123(14,3)$ & $7(19,4)$ & $116(14,1)$ & \\
\hline Outro Estado do Brasil & $110(12,8)$ & $6(16,7)$ & $104(12,6)$ & \\
\hline SEXO, $n(\%)$ & & & & \\
\hline Feminino & $560(65,1)$ & $32(88,9)$ & $528(64,1)$ & $0,002^{\mathrm{F}}$ \\
\hline Masculino & $300(34,9)$ & $4(11,1)$ & $296(35,9)$ & \\
\hline ESTADO CIVIL, $n(\%)$ & & & & \\
\hline Solteiro & $304(35,6)$ & $21(60)$ & $283(34,6)$ & $0,018^{Q}$ \\
\hline Casado & $476(55,7)$ & $14(40)$ & $462(56,4)$ & \\
\hline Viúvo & $20(2,3)$ & $0(0)$ & $20(2,4)$ & \\
\hline Outros & $54(6,3)$ & $0(0)$ & $54(6,6)$ & \\
\hline COR DA PELE, $n(\%)$ & & & & \\
\hline Branca & $207(24,8)$ & $8(22,9)$ & $199(24,9)$ & $0,867^{Q}$ \\
\hline Parda & $425(51)$ & $19(54,3)$ & $406(50,8)$ & \\
\hline Preta & $171(20,5)$ & $6(17,1)$ & $165(20,7)$ & \\
\hline Amarela & $29(3,5)$ & $2(5,7)$ & $27(3,4)$ & \\
\hline Indígena & $2(0,2)$ & $0(0)$ & $2(0,3)$ & \\
\hline Álcool, $n(\%)$ & $267(94,3)$ & $5(13,9)$ & $262(94,2)$ & $1,000^{\mathrm{F}}$ \\
\hline Tabagismo, $n(\%)$ & $40(14,1)$ & $1(20)$ & $39(14)$ & $0,536^{\mathrm{F}}$ \\
\hline Drogas Ilícitas, $n(\%)$ & $1(0,4)$ & $0(0)$ & $1(0,4)$ & $1,000^{\mathrm{F}}$ \\
\hline GRAU DE INSTRUÇÃO, $n(\%)$ & & & & \\
\hline Analfabeto & $7(0,8)$ & $0(0)$ & $7(0,9)$ & $0,925^{Q}$ \\
\hline Ensino Fundamental Incompleto & $32(3,8)$ & $1(2,9)$ & $31(3,8)$ & \\
\hline Ensino Fundamental Completo & $99(11,7)$ & $3(8,8)$ & $96(11,8)$ & \\
\hline Ensino Médio Completo & $447(52,7)$ & $20(58,8)$ & $427(52,4)$ & \\
\hline Ensino Superior Completo & $264(31,1)$ & $10(29,4)$ & $254(31,2)$ & \\
\hline
\end{tabular}

Legenda: $\mathrm{n}$ - frequência absoluta. \% - frequência relativa percentual. IIQ - intervalo interquartil. W - Teste de Mann-Whitney. Q Teste Qui-Quadrado de Pearson. F - Teste Exato de Fisher. Fonte: Autores.

De acordo com a classificação clínica da DF, foram subdivididos de acordo com os sintomas: Síndrome do Desconforto Pós Prandial (SDPP), Síndrome da Dor Epigástrica (SDE) e a apresentação concomitante entre as duas (SDPP e SDE). Assim, 10 (27,8\%) pacientes eram portadores apenas de SDE, 07 (19,4\%) apenas SDPP e 19 (52,8\%) referiam sintomas correspondentes às duas síndromes concomitantemente (Figura 1). 
Figura 1. Classificação clínica da Dispepsia Funcional de acordo com os sintomas. Aracaju 2018-2019.

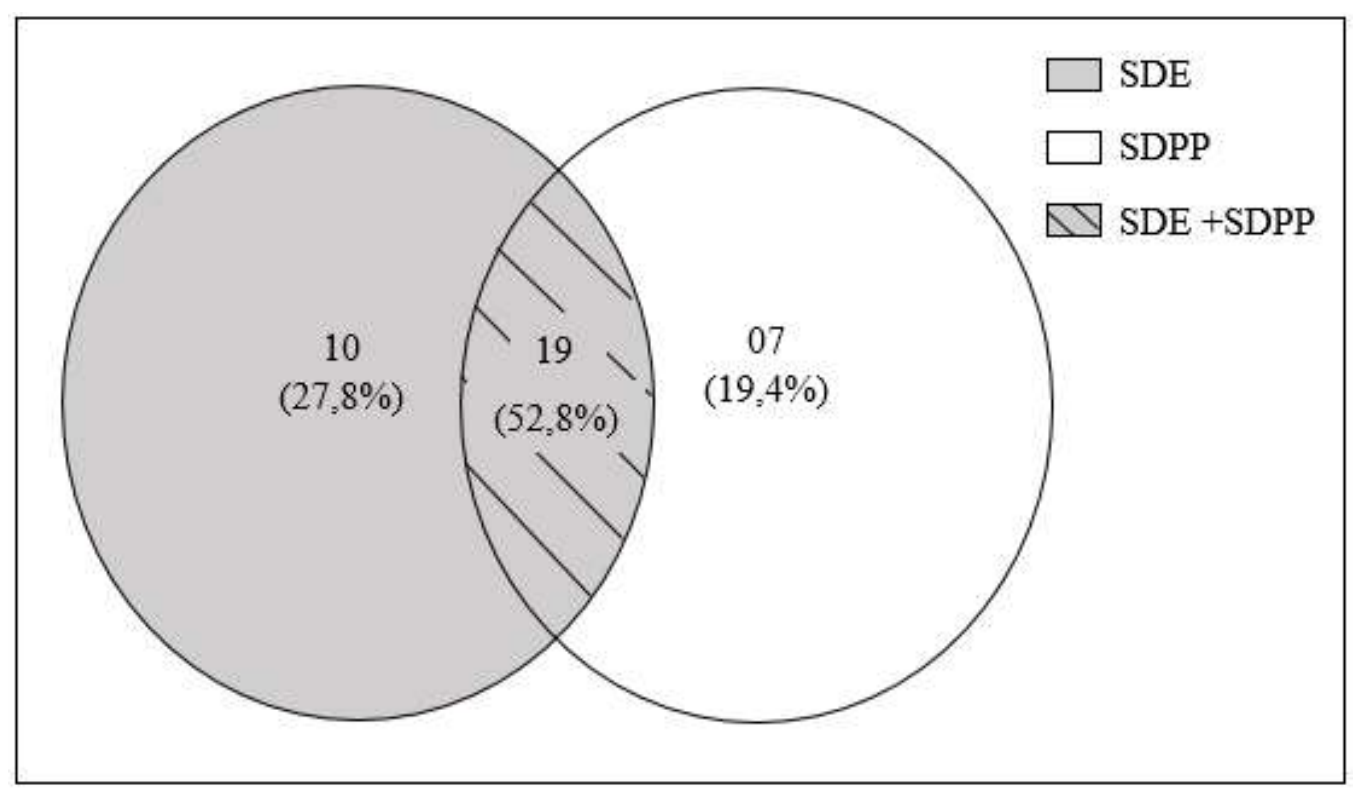

Fonte: Autores.

Dos pacientes com SDPP, ao somar o padrão isolado com o que corresponde à intersecção, 26 (72,2\%) se sentiam desconfortavelmente cheios (saciados) após uma refeição de tamanho habitual pelo menos 1 vez nos últimos 3 meses e desses, 10 há mais de 6 meses; 8 (22,2\%) referiram ser incapazes de terminar uma refeição de tamanho habitual pelo menos 1 vez nos últimos 3 meses e $5(62,5 \%)$ há mais de 6 meses.

Da mesma forma ao somar todos os entrevistados que se enquadraram na SDE com a intersecção, 29 (80,5\%) tiveram pelo menos uma vez no mês, nos últimos três meses, dor ou desconforto no meio do peito não relacionado a problemas cardíacos; $22(61,1 \%)$ manifestaram azia pelo menos 1 vez nos últimos três meses e 20 (57,1\%) apresentaram dor ou desconforto nos últimos três meses. Desses, 8 (42,1\%) referiram permanecer com essa dor por mais de seis meses; 18 (50\%) deles notaram que a dor ocorria e desaparecia completamente no mesmo dia, às vezes, nos últimos 3 meses. Quanto a intensidade dessa dor, 4 (20\%) pacientes a descreveram como suave, 10 (50\%) moderada, 5 (25\%) severa e 1 (5\%) muito severa. Questionados sobre o uso de antiácido para alívio, 4 (20\%) responderam nunca ou raramente notar alívio, 9 (45\%) reconheciam algum alívio e desses, 3 (15\%) referiam aliviar às vezes, $1(5 \%)$ muitas vezes, $2(10 \%)$ na maioria das vezes e 3 (15\%) sempre. Sete (35\%) deles não faziam uso de antiácidos.

Dentre os pacientes com queixa de dor epigástrica, quanto a frequência e fator de melhora, afirmaram que passava ou melhorava o sintoma após evacuação ou eliminação de gases: 6 (30\%) às vezes, 2 (10\%) na maioria das vezes e 4 (20\%) sempre. Identificaram também aliviar com movimentos ou trocas de posição do corpo: 8 (40\%) nunca ou raramente, 5 (25\%) às vezes, $1(5 \%)$ muitas vezes, $3(15 \%)$ maioria das vezes e $3(15 \%)$ sempre.

Queixavam-se que nos últimos seis meses a dor era constante no meio ou na área superior direita do seu abdome, 9 (25\%) pacientes e, quando questionados quanto a duração de mais de 30 minutos, responderam que: nunca ou raramente 3 $(27,3 \%)$, às vezes $1(9,1 \%)$, muitas vezes $2(18,2 \%)$, maioria das vezes $3(27,3 \%)$ e sempre $2(18,2 \%)$. Quanto ao aumento de intensidade dessa dor até ficar muito forte e contínua, os respondentes afirmaram: nunca ou raramente 3 (30\%) pacientes, às vezes 4 (40\%), muitas vezes $2(20 \%)$ e maioria das vezes 1 (10\%). Se essa dor desaparece entre os episódios, do seu aparecimento responderam que: nunca ou raramente 3 (30\%), ás vezes 3 (30\%), maioria das vezes 1 (10\%) e sempre 3 (30\%). Se essa dor impediu de realizar suas atividades usuais ou levou-o a ir urgentemente ao médico ou a um serviço de emergência, afirmaram que: nunca ou raramente $6(60 \%)$, às vezes $2(20 \%)$ e sempre $2(20 \%)$ (Tabela 2$).$ 
Tabela 2. Aspectos clínicos dos dispépticos funcionais segundo os critérios diagnósticos de Roma III. Aracaju, 2018 -2019.

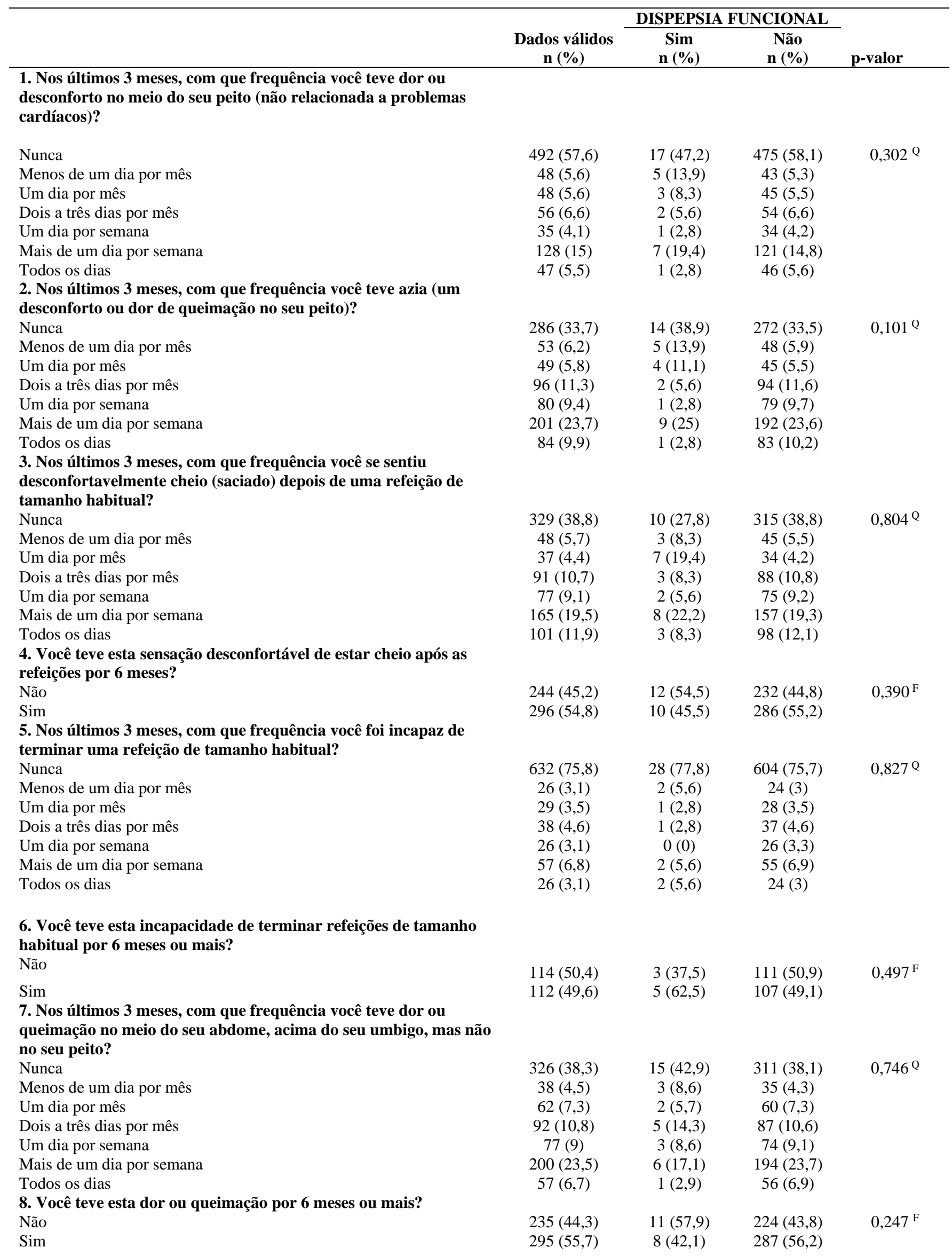


9. Esta dor ou queimação ocorre e depois desaparece

completamente durante o mesmo dia?

Nunca ou raramente

$\begin{array}{ccc}84(15,9) & 3(14,3) & 81(15,9) \\ & & \\ 131(24,8) & 4(19) & 127(25) \\ 34(6,4) & 3(14,3) & 31(6,1) \\ 96(18,1) & 3(14,3) & 93(18,3) \\ 184(34,8) & 8(38,1) & 176(34,6)\end{array}$

$0,631 \mathrm{Q}$

Às vezes

Muitas vezes

Maioria das vezes

Sempre

Muito severa

11. Essa dor ou queimação era aliviada com o uso de antiácidos?

Nunca ou raramente

Às vezes

Muitas vezes

Maioria das vezes

Sempre

Não uso antiácidos

12. Essa dor ou queimação normalmente melhorava ou passava após a evacuação ou eliminação de gases?

Nunca ou raramente

Às vezes

Muitas vezes

Maioria das vezes

Sempre

13. Com que frequência essa dor ou desconforto aliviou com movimentos ou trocas de posição do seu corpo?

Nunca ou raramente

Às vezes

Muitas vezes

Maioria das vezes

Sempre

14. Nos últimos 6 meses, com que frequência você teve dor

constante no meio ou na área superior direita do seu abdome?

Nunca

Menos de um dia por mês

Um dia por mês

Dois a três dias por mês

Um dia por semana

Mais de um dia por semana

Todos os dias

15. Esta dor durou 30 minutos ou mais?

Nunca ou raramente

Às vezes

Muitas vezes

Maioria das vezes

Sempre

16. Essa dor aumentou de intensidade até ficar muito forte e contínua?

Nunca ou raramente

Às vezes

Muitas vezes

Maioria das vezes

Sempre

17. Esta dor desapareceu completamente entre os episódios?

Nunca ou raramente

Às vezes

Muitas vezes

Maioria das vezes

Sempre

18. Essa dor o impediu de realizar suas atividades usuais ou levou-

$14(2,7)$

$123(23,4)$

$246(46,8)$

$108(20,5)$

$35(6,7)$

$82(15,5)$

$104(19,7)$

$30(5,7)$

$64(12,1)$

$127(24,1)$

$121(22,9)$

$275(52,3)$

$114(21,7)$

$17(3,2)$

$60(11,4)$

$60(11,4)$

$0(0)$

$4(20)$

$10(50)$

5 (25)

$1(5)$

$4(20)$

$3(15)$

$1(5)$

$2(10)$

3 (15)

7 (35)

$14(2,8)$

$119(23,5)$

$236(46,6)$

$103(20,4)$

$34(6,7)$

$78(15,4)$

$101(19,9)$

$29(5,7)$

$62(12,2)$

$124(24,4)$

$114(22,4)$

$294(55,7)$

$102(19,3)$

$28(5,3)$

$50(9,5)$

$54(10,2)$

$8(40)$

$6(30)$

$0(0)$

2 (10)

4 (20)

$267(52,8)$

$108(21,3)$

$17(3,4)$

$58(11,5)$

$56(11,1)$

$8(40)$

$5(25)$

$286(56,3)$

$97(19,1)$

$27(5,3)$

$1(5)$

$3(15)$

$47(9,3)$

$51(10)$

$545(64,3)$

$40(4,7)$

$42(5)$

$54(6,4)$

$42(5)$

$88(10,4)$

$37(4,4)$

$101(32,1)$

$68(21,6)$

$19(6)$

$49(15,6)$

$78(24,8)$

$117(37,4)$

$76(24,3)$

$34(10,9)$

$21(6,7)$

$65(20,8)$

$73(23,8)$

$84(27,4)$

$13(4,2)$

$23(7,5)$

$114(37,1)$
$519(63,8)$

$39(4,8)$

$40(4,9)$

$51(6,3)$

$41(5)$

$86(10,6)$

$37(4,6)$

$98(32,2)$

$67(22)$

$17(5,6)$

$46(15,1)$

$76(25)$

$114(37,6)$

$72(23,8)$

$32(10,6)$

$20(6,6)$

$65(21,5)$

$70(23,6)$

$81(27,3)$

$13(4,4)$

3 (30)

0 (0)

$22(7,4)$

$1(10)$

$111(37,4)$
$0,939^{\mathrm{Q}}$

$0,767^{Q}$

$0,502^{Q}$

$0,678^{Q}$

$0,710^{\mathrm{Q}}$

$0,287^{Q}$

$0,372^{Q}$

$0,979^{Q}$ o a ir urgentemente ver um médico ou ir a um serviço de emergência? 


\begin{tabular}{|c|c|c|c|c|}
\hline Nunca ou raramente & $201(64,4)$ & $6(60)$ & $195(64,6)$ & $0,262^{Q}$ \\
\hline Às vezes & $67(21,5)$ & $2(20)$ & $65(21,5)$ & \\
\hline Muitas vezes & $14(4,5)$ & $0(0)$ & $14(4,6)$ & \\
\hline Maioria das vezes & $14(4,5)$ & $0(0)$ & $14(4,6)$ & \\
\hline Sempre & $16(5,1)$ & $2(20)$ & $14(4,6)$ & \\
\hline
\end{tabular}

Legenda: $\mathrm{n}$ - frequência absoluta. \% - frequência relativa percentual. Q - Teste Qui-Quadrado de Pearson. F - Teste Exato de Fisher. Fonte: Autores.

Ao aplicar os questionários de Beck para diagnóstico de ansiedade e depressão notou-se que 19 (52,8\%) dentre os dispépticos funcionais preencheram os critérios para ansiedade e $9(25 \%)$ para depressão, sendo a forma leve a apresentação mais comum entre esses pacientes. (Tabela 3 ).

Tabela 3. Nível de ansiedade e depressão entre os dispépticos funcionais. Aracaju 2018-2019.

\begin{tabular}{lccc}
\hline & $\begin{array}{c}\text { Dados válidos } \\
\mathbf{n}(\boldsymbol{\%})\end{array}$ & $\begin{array}{c}\text { Sim } \\
\mathbf{n}(\boldsymbol{\%})\end{array}$ & $\begin{array}{c}\text { Não } \\
\mathbf{n}(\boldsymbol{\%})\end{array}$ \\
\cline { 3 - 4 } Ansiedade (BAI) & & & \\
Ausente & $472(54,9)$ & $17(47,2)$ & $455(55,2)$ \\
Leve & $214(24,9)$ & $11(30,6)$ & $203(24,6)$ \\
Moderado & $112(13,0)$ & $5(13,9)$ & $107(13,0)$ \\
Grave & $62(7,2)$ & $3(8,3)$ & $59(7,2)$ \\
Depressãor & & $667(80,9)$ \\
Mínimo & & $27(75)$ & $82(10)$ \\
Leve & $694(80,7)$ & $7(19,4)$ & $55(6,7)$ \\
Moderado & $89(10,3)$ & $1(2,8)$ & $20(2,4)$ \\
Grave & $56(6,5)$ & $1(2,8)$ & 0,236 \\
\hline
\end{tabular}

Legenda: $\mathrm{n}$ - frequência absoluta. \% - frequência relativa percentual. Teste Qui-Quadrado de Pearson. Fonte: Autores.

\section{Discussão}

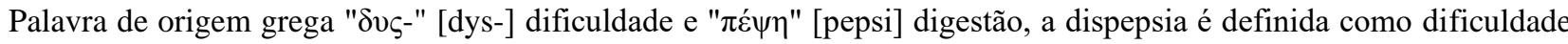
ou distúrbio da digestão, que é representada por um conjunto de sintomas relacionados ao trato gastrintestinal superior manifestando-se como dor, queimação ou desconforto, saciedade precoce e plenitude pós prandial. Contudo, para ser diagnosticado como dispepsia funcional, esse distúrbio não deve apresentar alterações na endoscopia digestiva alta e na leitura das biópsias, que justifiquem a queixa clínica (Oliveira, Fernandes, Silva, Lima, \& Brito, 2019). O estudo demonstrou que na população entrevistada apenas 36 (4,19\%) pacientes preencheram os critérios para o diagnóstico de Dispepsia Funcional (DF).

A DF é diagnosticada baseando-se no quadro clínico do paciente e na exclusão de outros diagnósticos. Tal conceito foi discutido pelo Consenso Roma III que definiu a síndrome dispéptica funcional como sendo a alteração clínica originada na região gastroduodenal associada à ausência de doença orgânica, sistêmica ou metabólica que a justifique. O Consenso Roma IV, lançado em 2016, reavaliou todos os critérios e manteve, quase sem alteração, os estabelecidos anteriormente pelo Consenso Roma III (Ferri, 2019). Apesar de terem sido aplicados os de Roma III, a análise dos sintomas e dos critérios diagnósticos para DF não foram prejudicadas, visto que a atualização não alterou diretamente a avaliação diagnóstica através deste instrumento.

As doenças do trato gastrointestinal superior, incluindo dispepsia, são comuns em adultos jovens e em idosos, embora a prevalência diminua com a idade acima de 60 anos (Walker \& Talley, 2019). Segundo Vargas et. al. (2016), o distúrbio funcional é a causa mais comum de dispepsia entre os jovens. Nesta pesquisa, a faixa etária se mostrou mais prevalente entre a segunda e a quarta década de vida, demonstrando maior frequência entre os adultos jovens.

Autores citam que sintomas dispépticos e azia são mais prevalentes na população urbana adulta brasileira (Almeida $e t$ al., 2017). Nossos dados revelaram que mais de $60 \%$ dos portadores de DF eram procedentes de Aracaju, maior centro urbano 
local. Nota-se também que são mais prevalentes no sexo feminino, ao considerar que as mulheres estão mais expostas a situações de estresse, tanto no âmbito social como também familiar, o que gera uma sobrecarga de atividades que as tornam mais suscetíveis ao aparecimento de diversos sintomas, incluindo os digestivos (Negre \& López, 2014; Sayuk \& Gyawali, 2020; Zelaya, Benítez, \& Turcios, 2020). No presente estudo, as mulheres representaram a grande maioria entre os portadores de dispepsia funcional.

Existem fatores de risco que são comuns nos dispépticos funcionais, seja naqueles que apresentam SDPP quanto nos portadores de SDE, tais como idade mais jovem e o estado civil solteiro, quando comparados aos demais (Fang et al., 2015). Em um levantamento brasileiro realizado por Almeida et al. (2017) com 548 indivíduos participantes, no qual mais da metade relatou queixa dispéptica, a maior frequência identificada foi entre os solteiros. Corroborando com este trabalho, nossos resultados também demonstraram uma alta prevalência entre os indivíduos solteiros, quando comparado aos casados, divorciados e viúvos. Observou-se também que a cor parda e os indivíduos com ensino médio completo foram mais prevalentes entre os portadores de DF.

Segundo Narváez e Gayoso-Cervantes (2016), o consumo de álcool e tabaco podem ser possíveis fatores de risco para dispepsia. Entretanto, não existe consenso quanto à associação de ingestão de álcool como um fator provocador ou não do quadro dispéptico (Duncanson, Talley, Walker, \& Burrows, 2017). O tabagismo, segundo Wei et al. (2020), é um fator de risco para alterações esofagogástricas estruturais, compatíveis com a dispepsia orgânica e não com a funcional. Embora não haja um padrão nessas relações, o presente estudo demonstrou que a minoria dos portadores de DF referiu fazer uso de bebida alcoólica e, um número menor, tabaco.

Do ponto de vista clínico, a DF pode ser classificada em dois grandes grupos segundo o ROMA IV: a síndrome do desconforto pós-prandial (SDPP) e a síndrome da dor epigástrica (SDE). De acordo com Caballero-Mateos e Cerezo (2018) a classificação surgiu para auxiliar na identificação e manejo da doença, em que agrupou os sintomas de empachamento pósprandial e a saciedade precoce na SDPP, e de dor e queimação epigástricas na SDE. Entretanto, é importante salientar que as duas síndromes podem aparecer concomitantemente, visto que podem ser apresentadas mutuamente e, mesmo que se sobreponham, não se excluem (Santos, 2019).

Um estudo realizado nos Estados Unidos da América, no Canadá e no Reino Unido, com 2100 indivíduos, mostrou que o subtipo mais comum da DF é a SDPP, atingindo mais que a metade da população de dispépticos estudada (Aziz et al., 2018). Em nossa casuística foi observada maior frequência do padrão clínico de SDE quando comparado com a SDPP. Contudo, a maioria dos dispépticos funcionais manifestaram, de forma mais frequente, os dois subtipos concomitantemente, ou seja, sintomas de desconforto pós prandial e de dor epigástrica paralelamente.

A presença de comorbidades em indivíduos com sintomas dispépticos e psiquiátricos é uma realidade comumente observada, com apresentação clínica e etiologia complexas (Trovão, 2020). Os pacientes com DF, num estudo realizado por Enck et al. (2017) com testes psicométricos, obtiveram maiores índices de ansiedade, depressão e somatização do que aqueles que não tinham sintomas digestivos. Essa relação evidencia o processamento patológico de estímulos viscerais associados a fatores psicossociais (Tshabalala, Tomita, \& Ramlall, 2019). Neste estudo, ao aplicar os inventários de Beck para ansiedade e depressão com intuito de avaliar a associação entre os sintomas dispépticos com transtornos psiquiátricos, evidenciou-se que mais da metade dos dispépticos funcionais referiram algum transtorno mental associado.

Os transtornos psiquiátricos, como depressão e ansiedade costumam aparecer frequentemente entre os pacientes com doenças gastrointestinais funcionais (Aro, Talley, Johansson, Agréus, \& Ronkainen, 2015). Alguns autores ainda sugerem que pacientes com síndrome do sofrimento pós-prandial e algum prejuízo na acomodação gástrica possuem uma maior chance de apresentar ansiedade (Talley, 2017). O presente estudo corrobora tais achados ao revelar que a maioria dos portadores de DF foram caracterizados como ansiosos, de acordo com o Inventário de Ansiedade de Beck (BAI), e 25\% com depressão, pelo 
Inventário de Beck para Depressão (BDI), confirmando a importante correlação entre doenças mentais e os sintomas gastrintestinais, principalmente nos dispépticos funcionais.

\section{Conclusão}

Evidenciou-se que o DF foi mais prevalente em mulheres, jovens e solteiras. A associação entre dispépticos funcionais com ansiedade e depressão, mostra-se como um possível componente envolvido na etiologia da DF. A presença dos sintomas de acordo com os critérios de diagnóstico de Roma III/IV apresentou uma predominância da SDE quando comparada com a SDPP, porém, a variante clínica mais comum foi a concomitante, ou seja, a que manifesta paralelamente tanto a SDE quanto a SDPP.

Portadores de dispepsia funcional, tendem a apresentar algum componente emocional associado às manifestações clínicas, o que requer novas pesquisas no sentido de aplicar questionários que avaliem não somente os aspectos psicológicos destes pacientes, mas também os níveis de estresse e de qualidade de vida, a fím de delinear melhor o perfil do dispéptico funcional e evidenciar formas mais eficazes de diagnóstico e conduta para esta síndrome cada vez mais comum em consultas gastroenterológicas.

\section{Agradecimentos}

Ao corpo integrante das clínicas participantes do estudo, a Gastroclínica, em especial à Sra. Irene Mendes Ribeiro Chaves e Joana Mendes Ribeiro Chaves Rezende e a Clínica do Aparelho Digestivo em nome da Dra Marta Maria Barbosa da Silva e Dr. Jilvan Pinto Monteiro.

\section{Referências}

Almeida, A. M., Martins, L. A. G., Cunha, P. L. T., Brasil, V. W., Félix, L. G. F., \& Passos, M. C. F. (2017). Prevalence of dyspeptic symptoms and heartburn of adults in Belo Horizonte, Brazil. Arquivos de Gastroenterologia. 54(1), 46-50. http://dx.doi.org/10.1590/s0004-2803.2017v54n1-09.

Aro P., Talley N. J., Johansson S. E., Agréus L., \& Ronkainen J. (2015). A ansiedade está ligada ao aparecimento de dispepsia na população sueca: um estudo de acompanhamento de 10 anos. Gastroenterology. 148(5), 928-937. Elsevier BV. http://dx.doi.org/10.1053/j.gastro.2015.01.039.

Aziz, I., Palsson, O., Tornblom, H., Sperber, A., Whitehead, W., \& Simrén, M. (2018). Epidemiology, clinical characteristics, and associations for symptombased Rome IV functional dyspepsia in adults in the USA, Canada, and the UK: a cross-sectional population-based study. The Lancet Gastroenterology \& Hepatology. 3(4), 252-262. http://dx.doi.org/10.1016/s2468-1253(18)30003-7.

Coelho, L. G. V., Marinho, J. R., Genta, R., Ribeiro, L. T., Passos, M. C. F., Zaterka, S., Assumpção, P. P., A Barbosa, A. J., Barbuti, R., \& Braga, L. L. (2018). IVth Brazilian consensus conference on helicobacter pylori infection. Arquivos de Gastroenterologia. 55(2), 97-121. http://dx.doi.org/10.1590/s00042803.201800000-20.

Caballero-Mateos, A. M., \& Cerezo, E. R. (2018). Dyspepsia, functional dyspepsia and Rome IV criteria. Revista Española de Enfermedades Digestivas. 110, 530-531. http://dx.doi.org/10.17235/reed.2018.5599/2018.

Drossman, D. A. (2016). Functional Gastrointestinal Disorders: History, Pathophysiology, Clinical Features and Rome IV. Gastroenterology. 150(6), 12621279. http://dx.doi.org/10.1053/j.gastro.2016.02.032.

Duncanson, K. R., Talley, N. J., Walker, M. M., \& Burrows, T. L. (2017). Food and functional dyspepsia: a systematic review. Journal Of Human Nutrition And Dietetics. 31(3), 390-407. http://dx.doi.org/10.1111/jhn.12506.

Enck, P., Azpiroz, F., Boeckxstaens, G., Elsenbrunch, S., Feinle-Bisset, C., Holtmann, G., Lackner, J. M., Ronkainen, J. Schemann, M., \& Stengel, A. (2017). Functional dyspepsia. Nature Reviews Disease Primers. 3(1), 1-20. http://dx.doi.org/10.1038/nrdp.2017.81.

Fang, Y. J., Liou J. M., Chen, C. C., Lee, J. Y., Hsu, Y. C., \& Chen, M. J. (2015). Distinct aetiopathogenesis in subgroups of functional dyspepsia according to the Rome III criteria. Gut. 64(10), 1517-1528.

Ferreira, I. K., Bello, S. R.de B., \& Gomes, K. S. G. (2018). O tratamento da dispepsia funcional na atenção primária à saúde. Revista de Saúde Pública do Paraná. 1(1), 116-122. http://dx.doi.org/10.32811/2595-4482.2018v1n1.50.

Ferri, M. K. (2019). Potencial papel de fatores genéticos nos sintomas da dispepsia funcional: estudo caso-controle. Dissertação (Mestrado) - Curso de Medicina. Universidade Federal do Rio Grande do Sul, Porto Alegre. 
Harer, K. N; Hasler, L. H. (2020). Functional Dyspepsia: A Review of the Symptoms, Evaluation, and Treatment Options. Gastroenterology \& Hepatology. Michigan, 16(2), 66-74.

Mayer, E. A., Labus, J. S., Tillisch, K., Cole, S. W., \& Baldi, P. (2015). Towards a systems view of IBS. Nat Rev Gastroenterol Hepatol, 12(10), 592-605. 10.1038/nrgastro.2015.121

Moloney, R. D., Johnson, A. C., O'Mahony, S. M., Dinan, T. G., Greenwood-Van Meerveld, B., \& Cryan, J. F. (2016). Stress and the Microbiota-Gut-Brain Axis in Visceral Pain: Relevance to Irritable Bowel Syndrome. CNS Neurosci Ther, 22(2), 102-117. 10.1111/cns.12490

Narváes, D. R. V., \& Gayoso-Cervantes, M. (2016). Estrés laboral y su correlación con la prevalencia de dispepsia funcional en militares en actividad que acudieron al hospital geriátrico del ejército. Revista de Gastroenterologia do Peru. 1(37), 16-21.

Negre, M. M. M., \& López, M. R. (2014). Functional dyspepsia: clinical-epidemiological and endoscopic characterization. Revista de Ciências Médicas, $5(18), 733-742$

Oliveira, S. M. de, Fernandes, W. A. A. de B., Silva, A. S., Lima A. V. F. da S., \& Brito, F. M. de. (2019). Características Clínicas e Fatores De Risco Da Dispepsia Funcional Em Pessoas Idosas: revisão integrativa. VI Congresso Internacional de Envelhecimento Humano. 6(1), 1-12.

Santos, I. B. (2019). Contagem de eosinófilos na segunda porção duodenal em pacientes com dispepsia funcional. 106 f. Tese (Doutorado) - Curso de Medicina. Faculdade de Medicina da Universidade de São Paulo, São Paulo.

Stanghellini, V., Chan, F. K. L., Hasler, W. L., Malagelada, J. R., Suzuki, H., Tack, J., \& Talley, N. J. (2016). Gastroduodenal Disorders. Gastroenterology. 150(6), 1380-1392. http://dx.doi.org/10.1053/j.gastro.2016.02.011.

Sayuk, G. S., \& Gyawali, C. P. (2020). Functional Dyspepsia: diagnostic and therapeutic approaches. Drugs. 80(13), 1319-1336. http://dx.doi.org/10.1007/s40265-020-01362-4.

Talley, N. J. (2017). Functional Dyspepsia: advances in diagnosis and therapy. Gut And Liver. 11(3), 349-357. http://dx.doi.org/10.5009/gnl16055.

Trovão, J. N. da N. A. S. (2020). Inovação e Intervenção em Perturbações Funcionais Gastrointestinais. Dissertação (Mestrado) - Curso de Medicina. Universidade do Porto, Porto.

Tshabalala, S. J., Tomita, A., \& Ramlall, S. (2019). Depression, anxiety and stress symptoms in patients presenting with dyspepsia at a regional hospital in KwaZulu-Natal province. South African Journal Of Psychiatry, 25, 17. http://dx.doi.org/10.4102/sajpsychiatry.v25i0.1382.

Vargas, M., Talledo-Ulfe, L., Samaniego, R., Heredia, P., Rodriguez, C. A. S., Mogollón, C. A., Enriquez, W. F., \& Meija, C. R. (2016). Dispepsia funcional en estudiantes de ocho facultades de medicina peruanas. Influencia de los hábitos. Acta Gastroenterológica Latinoamericana. 46(2), 95-101.

Vinaccia, S., Quiceno, J. M., Medina, A., Castañeda, B., \& Castelblanco, P. (2014). Calidad de vida, resiliencia, percepción y conducta de enfermedad en pacientes con diagnóstico de dispepsia funcional. Acta Colombiana de Psicología. 61-70. Editorial Universidad Catolica de Colombia. http://dx.doi.org/10.14718/acp.2014.17.1.7.

Von, R. P. S., Mazzoleni, L. E., Sander, G. B., \& Francisconi, C. F. M. (2010) Portuguese validation of the Rome III Diagnostic Questionnaire for Functional Dyspepsia Arq Gastroenterol. 47(4).

Walker, M. M., \& Talley, N. J (2019). Functional Dyspepsia in the Elderly. Current Gastroenterology Reports. 21(10), 1-6. http://dx.doi.org/10.1007/s11894019-0722-5.

Wei, Z. C., Yang, Q., Yang, Q., Yang, J., Tantai, X. X., Xing, X., Xiao, C. L., Pan, Y. L., Wang, J. H., \& Liu, N. (2020). Predictive value of alarm symptoms in patients with Rome IV dyspepsia: a cross-sectional study. World Journal Of Gastroenterology. $26(30)$, 4523-4536. http://dx.doi.org/10.3748/wjg.v26.i30.4523.

Zelaya, R. S. D., Benítez, C. E. C., \& Turcios, N. M. R. (2020). Síndrome dispéptico en los pacientes que consultan la UCSF-I Conchagua. Crea Ciencia Revista Científica. 13(1), 11-21.. http://dx.doi.org/10.5377/creaciencia.v13i1.10462. 